\title{
Adaptive Temporal Triplet-loss for Cross-modal Embedding Learning
}

\author{
David Semedo \\ NOVA LINCS \\ Universidade NOVA de Lisboa, Portugal \\ df.semedo@campus.fct.unl.pt
}

\author{
João Magalhães \\ NOVA LINCS \\ Universidade NOVA de Lisboa, Portugal \\ jm.magalhaes@fct.unl.pt
}

\section{Static margin over time}

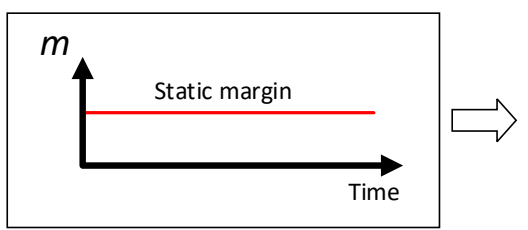

(a) Lack expressiveness for transposing temporal correlations to embedding space
Temporal smoothing

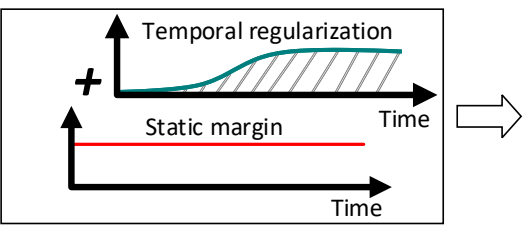

(b) Directly enforces embedding properties using temporal regularization
Adaptive temporal margins

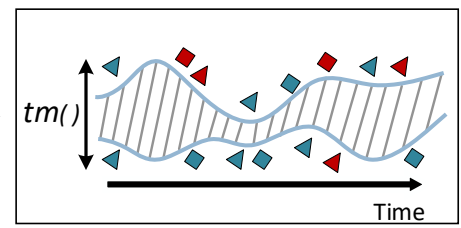

(c) Samples are adaptively structured according to temporal correlation

Figure 1: Margins as function of time in the triplet loss function.

\begin{abstract}
There are many domains where the temporal dimension is critical to unveil how different modalities, such as images and texts, are correlated. Notably, in the social media domain, information is constantly evolving over time according to the events that take place in the real world [21, 32, 42]. In this work, we seek for highly expressive loss functions that allow the encoding of data temporal traits into cross-modal embedding spaces. To achieve this goal, we propose to steer the learning procedure of such embedding through a set of adaptively enforced temporal constraints. In particular, we propose a new formulation of the triplet loss function, where the traditional static margin is superseded by a novel temporally adaptive maximum margin function. This novel redesign of the static margin formulation, allows the embedding to effectively capture not only the semantic correlations across data modalities, but also data's fine-grained temporal correlations. Our experiments confirm the effectiveness of our model in structuring different modalities, while organizing data according to temporal correlations. Moreover, we experimentally highlight how can these embeddings be used for multimedia understanding.
\end{abstract}

\section{CCS CONCEPTS}

- Information systems $\rightarrow$ Multimedia and multimodal retrieval; • Computing methodologies $\rightarrow$ Neural networks;

Permission to make digital or hard copies of all or part of this work for personal or classroom use is granted without fee provided that copies are not made or distributed for profit or commercial advantage and that copies bear this notice and the full citation on the first page. Copyrights for components of this work owned by others than the author(s) must be honored. Abstracting with credit is permitted. To copy otherwise, or republish, to post on servers or to redistribute to lists, requires prior specific permission and/or a fee. Request permissions from permissions@acm.org.

MM '20, October 12-16, 2020, Seattle, WA, USA

( 2020 Copyright held by the owner/author(s). Publication rights licensed to the Association for Computing Machinery.

ACM ISBN 978-1-4503-7988-5/20/10 . \$15.00

https://doi.org/10.1145/3394171.3413540

\section{KEYWORDS}

adaptive temporal triplet-loss; temporal cross-modal embeddings; cross-modal embeddings; multimedia-understanding.

\section{ACM Reference Format:}

David Semedo and João Magalhães. 2020. Adaptive Temporal Triplet-loss for Cross-modal Embedding Learning. In Proceedings of the 28th ACM International Conference on Multimedia (MM '20), October 12-16, 2020, Seattle, WA, USA. ACM, New York, NY, USA, 10 pages. https://doi.org/10.1145/3394171. 3413540

\section{INTRODUCTION}

Time plays a key role in shaping the semantics of a multimodal instance, by defining its temporal context. This has motivated research on rich modality representations - embeddings - that not only capture the joint intricacies of individual modalities and how they are correlated, but also incorporate external temporal information to aid the structuring of such embeddings. These embeddings are of utmost importance for addressing downstream multimedia understanding tasks, such as summarization [34, 48, 51], visual question-answering [2, 24, 33] (VQA) and image captioning [18, 44], to manipulate different modalities interchangeably. For example, finding the images or texts that best summarizes a text/image (summarization), finding the best answer during a conversational session, whether it is an image, a text, or both.

Cross-modal embeddings [9, 10, 29, 30, 45, 46, 50], leverage on multimodal machine learning $[3,26]$ and metric learning [20], to learn a common embedding space that structures images and their textual descriptions based on their correlations, thus bridging heterogeneous representations. Static cross-model embeddings that use the triplet loss function, apply a static margin which does not allow to encode temporal information, Figure 1(a). Taking a step forward towards incorporating time information in cross-modal embedding spaces, in [36,37], an approach was proposed to structure a cross-modal embedding space based on semantic (category information) and time information. To incorporate time, the optimization 
framework was formulated using the standard triplet-loss [35], that organizes instances based on their categories, and then a smoothing term is applied to penalize the model when two temporally correlated instances from the same category are far away, Figure 1(b). This smoothing term provides a weak signal to the model, w.r.t. how it should improve. Namely, while it signals errors in the embedding structuring, it fails to provide accurate information regarding how should the model improve.

In this paper, we move beyond this simple smoothing term [37], Figure 1(b), and propose a novel differentiable adaptive temporal triplet-loss formulation, Figure 1(c), under a neural embedding learning framework, for temporal cross-modal embedding learning. Our approach builds upon the hypothesis of adapting the triplet-loss margin during training for higher expressiveness of the embedding learning. In particular, we depart from previous work, by formulating adaptive and triplet-specific temporal margins, that capture how correlated in time two instances are, and provide this information directly to the model. As the margin of each triplet will be directly quantifying temporal correlation between two instances, it results in more accurate gradient for the optimization process that explicitly tells the model how it should be updated to correct the distance between two instances in the embedding space. This can be seen as providing specific information to the model, releasing it from having to guess how far apart the instances should be.

In summary, the key contributions of this paper are:

(1) A neural framework for temporal cross-modal embedding learning, supporting a fine-grain structuring of the embedding space, to cope with data complex temporal correlations;

(2) An expressive adaptive temporal triplet-loss formulation, that enables an effective joint structuring of both data modalities (image+text) and contextualizing information, in a common cross-modal space;

(3) A thorough analysis of the proposed approach, comprised by a canonical demonstration and evaluation of what the proposed model accomplishes, evidencing the characteristics of the proposed embedding.

The proposed adaptive temporal triplet-loss was designed to incorporate time, however, it can be easily generalized to accommodate other types information such as location or other metadata.

\section{RELATED WORK}

Several works model temporal data based on time-series methods $[23,31,49,52]$, where data are sequences of observations, that are close in time and usually collected at a constant rate. This is the case of joint representation learning methods for video [17, 27, 41, 53], that are characterized by temporally aligned visual and audio modalities, in which models that capture sequence dependencies over time steps (e.g. RNNs) are commonly adopted. In contrast, for timestamped multimodal data, instances come from different and independent sources (e.g. users) that capture the same event at different instants in time, e.g. two medical images of the same patient, three weeks apart, or pictures of a race crash published by different users. Modeling temporal aspects of heterogeneous data, has been studied in many areas [12, 19, 22, 25, 32, 36, 42, 43, 54]. Kim and Xing [19] tackled the image retrieval problem from a time-sensitive perspective and proposed a multi-task regression on multivariate point processes, where the multivariate point process correspond to the image streams. Uricchio et al. [43] studied the hypothesis of using temporal information (e.g. tag frequency over time) to improve image annotation and retrieval on social media content. The fact that some concepts' time-series revealed to be correlated with Google Search trends, evidences the importance of temporal aspects for such tasks. Tsytsarau et al. [42] model the dynamics of news events and social media reactions to those events, to reveal how users' sentiment changes over time. In [5] a dynamic topic model is proposed to capture temporal behaviour of words based on the evolution of their interactions over time. By treating elements as symbols, topic model approaches lack all the properties of distributed representations [4]. Hamilton et al. [12] and Yao et al. [54] proposed diachronic word embeddings, capturing and encoding the temporal evolution of individual word's context, by factorizing a point-wise mutual information matrix at each timestep. Then a smoothing term is added to the objective function to align embeddings. Similar research has been done in diachronic crossmodal settings [39] to capture how the correlations between the two modalities evolve over time.

A wide range of static cross-modal embeddings [9, 10, 29, 30, $45,50]$ have been proposed. Neural network methods are the most widely adopted approach, with projections being learned end-toend. Autoencoders are an example of such approach $[6,10]$ which learns a common cross-modal embedding minimizing the reconstruction error. Yan and Mikolajczyk [50] explored a neural archictecture to approximate the CCA algorithm and proposed the Deep Canonical Correlation Analysis (Deep-CCA) to match images and text. Peng et al. [28] took a step further by adding extra constraints over inter-modal instance relations, instead of focusing in pairwise visual-textual correlation. Fan et al. [9], propose a neural network fusion approach to combine global convolutional network features with image descriptions to obtain a richer semantic embedding space. In [45] a projection network and a modality classifier are jointly learned under an adversarial learning setting. In [29] the authors model intra and inter modality correlations, to unveil complex modality interactions. Image patches were used to form visualtextual pairs, allowing for the unveiling of more fine-grained latent correlations.

Recently, Semedo and Magalhaes [38] proposed a scheduled adaptive margin formulation for triplet loss, achieving state-ofthe-art performance on cross-modal retrieval. Also, He et al. [14] adapted the quadruplet loss function [7], to the cross-modal problem. The proposed loss function uses two triplet functions to cover two possible permutations between two positive instances and a negative instance. In its most generic formulation, this approach can be seen as enforcing two distinct aspects in the embedding structuring, what suits our needs: modeling semantic and temporal correlation. Building on previous evidence on the importance of temporal signals [22, 32, 42], we depart from the standard tripleloss formulation and, inspired by the adaptive triplet loss [38] and quadruplet loss [14] formulations, we propose a novel formulation of a temporal cross-modal embedding learning. Previous work in this same vein used temporal constraints over standard triplet loss [37]. In contrast to previous work, we do not use static margins and instead propose a well grounded theoretical formulation of the triplet loss with adaptive temporal margins. 


\section{TEMPORAL CROSS-MODAL EMBEDDINGS}

In this paper we are interested in a class of problems dealing with a set $C$ of timestamped instances $d^{i}=\left(x_{V}^{i}, x_{T}^{i}, t^{i}\right) \in C$, where without loss of generality, $x_{V}^{i} \in X_{V} \subseteq \mathbb{R}^{D_{V}}$ and $x_{T}^{i} \in X_{T} \subseteq \mathbb{R}^{D_{T}}$ are the instance's image and text feature representations, respectively, and $t^{i}$ the instance's timestamp (please note that $d^{i}$ may be comprised of any two modalities, not necessarily image and text). The task is additionally constrained when category labels are introduced: given a set of semantic categories $L=\{$ crash, show, ... $\}$, each instance $d^{i} \in C$ is associated to one or more categories $l^{i}=\left\{l_{j}^{i}\right\}_{j=1}^{\left|l^{i}\right|}$, such that $l_{j}^{i} \in \boldsymbol{L}$. Category information enables going beyond image-text pairwise correlations, by requiring data to be organized not solely by cross-modality correlations, but also by category.

Original modality feature spaces $X_{V}$ and $X_{T}$ are heterogeneous - having distinct semantics and distributions that are correlated over time. The goal is to obtain a common embedding space, where temporal correlations are used, together with semantic correlations, to structure the embedding space. Therefore, a temporal crossmodal embedding space can be defined as follows:

Definition 1. A temporal cross-modal embedding refers to a common embedding space $\mathcal{S} \in \mathbb{R}^{D}$, learned from a timestamped collection $C$, that structures visual and textual elements of data instances according to their semantic categories $l^{i}$ and pairwise temporal correlations, measured by a function $f_{t}(\cdot)$, across different modalities.

The original representations, of different modalities, are projected into the common embedding space $\mathcal{S}$ with auxiliary projection functions:

$$
\mathcal{P}_{\boldsymbol{\theta}_{V}}(\cdot): X_{V} \mapsto \mathcal{S} \quad \mathcal{P}_{\boldsymbol{\theta}_{T}}(\cdot): X_{T} \mapsto \mathcal{S}
$$

where $X_{V} \subseteq \mathbb{R}^{D_{V}}$ and $X_{T} \subseteq \mathbb{R}^{D_{T}}$ are the original modalities' feature spaces. The aforementioned projections map images $x_{V}$ and texts $x_{T}$ to the new temporal cross-modal embedding, $\mathcal{S} \subseteq \mathbb{R}^{D}$. Projections are $\ell_{2}$ normalized, thus, similarity between any two projected instance's modalities $x^{i}$ and $x^{j}$, is computed as the dot product (cosine similarity),

$$
s\left(x^{i}, x^{j}\right)=\mathcal{P}_{\boldsymbol{\theta}}\left(x^{i}\right) \cdot \mathcal{P}_{\boldsymbol{\theta}}\left(x^{j}\right) .
$$

From now on, let $* \in\{V, T\}$, on the remainder of this paper, to avoid notation cluttering.

In the collection $C$, the temporal correlation between any two instances $d^{i}$ and $d^{j}$, is expressed by a function $f_{t}\left(d^{i}, d^{j}\right)$, in the interval $[0,1]$, where 1 denotes maximal correlation and 0 no correlation. Function $f_{t}$ can follow different temporal distributions. We now state the fundamental properties of the proposed embedding space that projections $\mathcal{P}_{\boldsymbol{\theta}_{V}}(\cdot)$ and $\mathcal{P}_{\boldsymbol{\theta}_{T}}(\cdot)$ need to satisfy:

Property 1. If $d^{i}$ and $d^{j}$ belong to the same semantic category, their similarity must be high, i.e. $s\left(x_{*}^{i}, x_{*}^{j}\right)>r_{1}$, with $r_{1} \in \mathbb{R}$. Otherwise, their similarity must be minimal, and $s\left(x_{*}^{i}, x_{*}^{j}\right) \ll r_{1}$.

Property 2. Temporally uncorrelated instances, i.e. $f_{t}\left(d^{i}, d^{j}\right) \rightarrow$ 0 , of the same semantic category $l^{j}$, are expected to lie in the same coarse-grained neighborhood: $r_{0}>s\left(x_{*}^{i}, x_{*}^{j}\right)>r_{1}$, with $r_{0} \in \mathbb{R}$;

Property 3. Temporally correlated instances, i.e. $f_{t}\left(d^{i}, d^{j}\right) \rightarrow 1$, of the same semantic category $l^{j}$, will lie in the same fine-grained neighborhood: $s\left(x_{*}^{i}, x_{*}^{j}\right)>r_{0}$, with $r_{0}>r_{1}$.

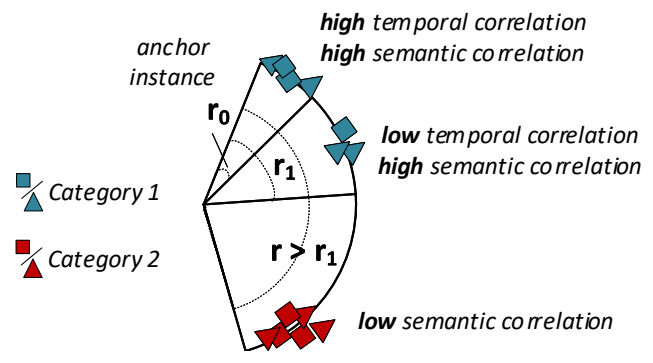

Figure 2: Properties illustration over the unit circle.

These properties are illustrated in Figure 2 through different neighborhoods in the embedding space. Note that each $r$ denotes similarity, meaning that if $r$ is high, the angle is small.

\section{TEMPORAL CROSS-MODAL PROJECTIONS}

The learning of the projection functions $\mathcal{P}_{\boldsymbol{\theta}_{V}}(\cdot)$ and $\mathcal{P}_{\boldsymbol{\theta}_{T}}(\cdot)$, needs to learn how to embed data by accounting for both semantic and temporal dimensions. This requires capturing the temporal traits of data, within each semantic category. To jointly accommodate the two dimensions, we propose a two-fold approach:

Semantic dimension - structure modality projections such that semantic correlation (based on semantic categories) is preserved, by being maximized in the new embedding space (Property 1);

Temporal dimension - perform embeddings' structuring for instances of the same category, according to pairwise temporal correlations (Properties 2 and 3).

The two aspects are covered by two jointly optimized loss functions $\mathcal{L}_{s}$ and $\mathcal{L}_{t}$, that enforce the semantic and temporal dimension requirements, respectively. Such projections are learned by minizing the following objective function

$$
\underset{\boldsymbol{\theta}_{V}, \boldsymbol{\theta}_{T}}{\arg \min } \mathcal{L}\left(\boldsymbol{\theta}_{V}, \boldsymbol{\theta}_{T}\right)=\underset{\boldsymbol{\theta}_{V}, \boldsymbol{\theta}_{T}}{\arg \min } \mathcal{L}_{s}\left(\boldsymbol{\theta}_{V}, \boldsymbol{\theta}_{T}\right)+\mathcal{L}_{t}\left(\boldsymbol{\theta}_{V}, \boldsymbol{\theta}_{T}, \boldsymbol{\theta}_{\text {temp }}\right),
$$

where $\mathcal{L}_{s}$ corresponds to a cross-modal loss that maximizes projections' similarity for semantically similar instances and minimizes similarity otherwise. The $\mathcal{L}_{t}$ loss corresponds to a novel adaptive temporal triplet-loss, that temporally structures embeddings of instances of the same category, according to a set of temporal restrictions. The temporal restrictions are responsible for enforcing Properties 2 and 3 , under a temporal distribution $\boldsymbol{\theta}_{\text {temp }}$. Both $\boldsymbol{\theta}_{V}$ and $\theta_{T}$, shared across the two losses, correspond to image and text projections learnable weights, respectively. The global loss $\mathcal{L}(\cdot)$ captures semantic and temporal correlations at inter-category, inter-modality and inter-instance granularity levels. We will refer to our model as TXS-Adapt.

\subsection{Semantic Maximum-Margin Loss}

Target projection functions $\mathcal{P}_{\boldsymbol{\theta}_{V}}$ and $\mathcal{P}_{\boldsymbol{\theta}_{T}}$ map instance representations, on the corresponding original spaces, to $\mathcal{S}$. According to Property 1, projections of instances that share at least one category should be similar, otherwise, they should be dissimilar. To this extent, we formulate $\mathcal{L}_{s}$ using triplet-loss [35]. Namely, given 


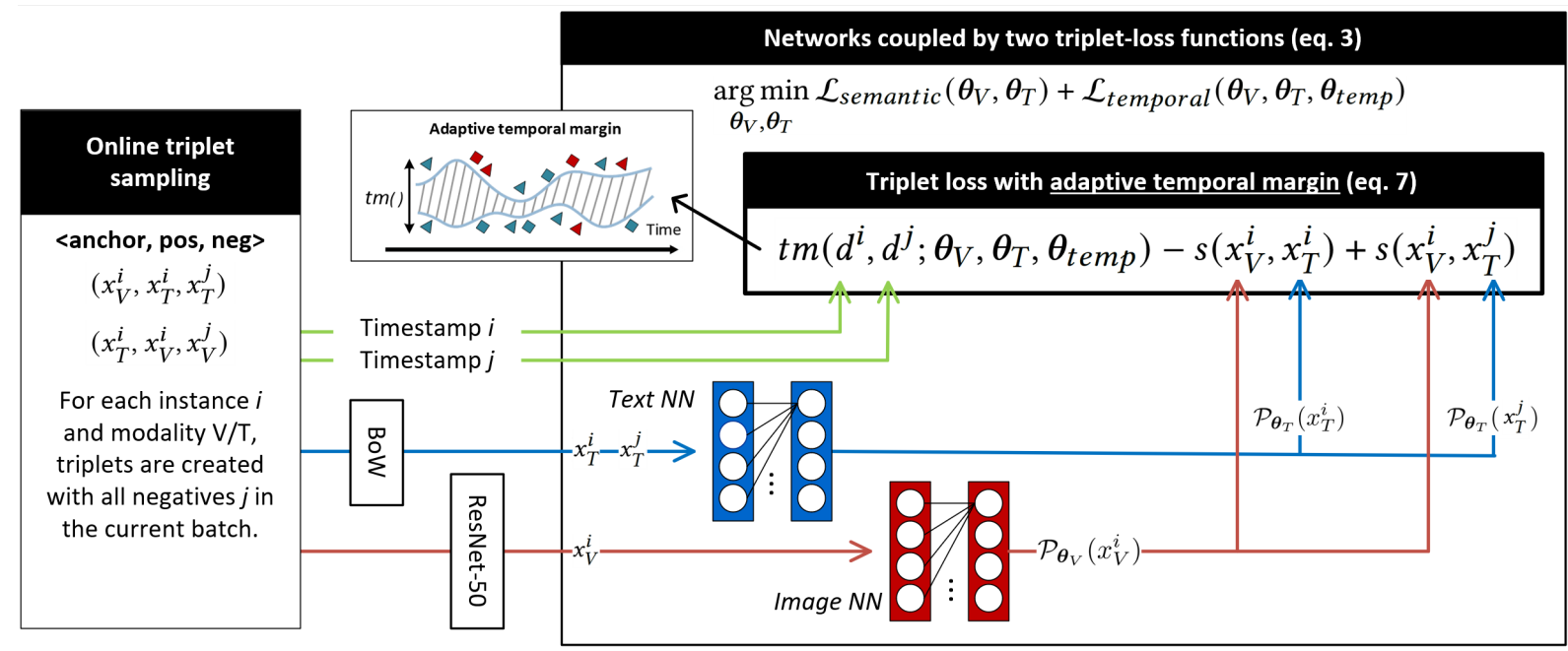

Figure 3: Architecture overview. Triplets are sampled covering all instances $i$ and modalities, from the current batch.

a triplet $\left(x_{*}^{i}, x_{*}^{p}, x_{*}^{n}\right)$, similarity between a instance $x_{*}^{i}$ and a positive (sharing at least one semantic category) instance $x_{*}^{p}$, must be greater than the similarity of $x_{*}^{i}$ and a negative instance $x_{*}^{n}$, by at least a margin $m$ :

$$
s\left(x_{*}^{i}, x_{*}^{p}\right)>s\left(x_{*}^{i}, x_{*}^{n}\right)+m .
$$

Equation 4 can be relaxed to a differentiable function using the hinge loss [15]. Accordingly, we define $\mathcal{L}_{s}$ by enforcing an instance of eq. 4 constraint for each instance $d^{i}$ as:

$$
\begin{aligned}
\mathcal{L}_{s}\left(\boldsymbol{\theta}_{V}, \boldsymbol{\theta}_{T}\right) & =\sum_{i, n}\left[m-s\left(x_{V}^{i}, x_{T}^{i}\right)+s\left(x_{V}^{i}, x_{T}^{n}\right)\right]_{+} \\
& +\sum_{i, n}\left[m-s\left(x_{T}^{i}, x_{V}^{i}\right)+s\left(x_{T}^{i}, x_{V}^{n}\right)\right]_{+},
\end{aligned}
$$

where $[x]_{+}$denotes the function $\max (0, x), x_{V}^{n}, x_{T}^{n}$ are images and texts feature representations from negative instances, and $m=$ 1 the margin. Cross-modality semantic correlations are captured by enforcing constraints in the image to text and text to image directions.

\subsection{Adaptive Temporal Maximum-Margin Loss}

Temporal structuring between instances of the same semantic category is governed by Property 2 and Property 3, which are established on a pairwise basis, i.e. between every two instances $d^{i}$ and $d^{j}$. Thus, we enforce each property as a temporal constraint between two instances. The constraint-based maximum margin approach from eq. 5 adopts a static margin $m$ to structure instances based on their semantic categories. In order to model both finegrain and coarse-grain pairwise temporal correlations, we cannot enforce a single, static margin, otherwise, only two levels of correlation could be captured: not correlated (0) or correlated (1). To overcome this, we propose an adaptive temporal triplet-loss, where the static margin $m$ from eq. 4 is replaced by an adaptive temporal margin function $\operatorname{tm}(\cdot)$, enforcing a pairwise specific margin, for each pair of instances of the same category:

$$
s\left(x_{V / T}^{i}, x_{T / V}^{i}\right)>s\left(x_{V / T}^{i}, x_{T / V}^{j}\right)+\operatorname{tm}\left(d^{i}, d^{j}\right) .
$$

The function $\operatorname{tm}(\cdot)$ is used to guide the structuring of the corresponding instances by the neural model, according to their pairwise temporal correlations. The magnitude of $\operatorname{tm}(\cdot)$ is defined according to a set of temporal constraints. These constraints will reveal how badly structured the instances are w.r.t. to each other, according to their temporal correlations. The signal that comes from the magnitude of $\mathrm{tm}(\cdot)$ explicitly provides strong supervision to the model regarding how far the two instances should be.

Given an adaptive margin function $\operatorname{tm}\left(d^{i}, d^{j}\right)$, we define $\mathcal{L}_{t}$ as follows:

$$
\begin{aligned}
& \mathcal{L}_{t}\left(\boldsymbol{\theta}_{V}, \boldsymbol{\theta}_{T}, \boldsymbol{\theta}_{\text {temp }}\right)= \\
& \quad \sum_{i, j}\left[t m\left(d^{i}, d^{j} ; \boldsymbol{\theta}_{V}, \boldsymbol{\theta}_{T}, \boldsymbol{\theta}_{\text {temp }}\right)-s\left(x_{V}^{i}, x_{T}^{i}\right)+s\left(x_{V}^{i}, x_{T}^{j}\right)\right]_{+} \\
& +\sum_{i, j}\left[t m\left(d^{i}, d^{j} ; \boldsymbol{\theta}_{V}, \boldsymbol{\theta}_{T}, \boldsymbol{\theta}_{\text {temp }}\right)-s\left(x_{T}^{i}, x_{V}^{i}\right)+s\left(x_{T}^{i}, x_{V}^{j}\right)\right]_{+} .
\end{aligned}
$$

To model the adaptive temporal margin $\operatorname{tm}(\cdot)$, we define two competing restrictions $R_{a}$ and $R_{b}$, capturing properties 2 and 3, respectively. These restrictions will be defined as trade-offs between temporal correlation and projections' similarity. Consider the diagram on Figure 4, illustrating the constraints rationale. In particular, temporal correlations are expressed by the function $f_{t}(\cdot)$ based on data timestamps and the cross-modal projections similarity is expressed by the function $f_{s}(\cdot)$ that evaluates how similar any two projected instances are.

The first restriction $R_{a}$ will state that if two instances are temporally uncorrelated, then their projections should be made dissimilar by $\mathcal{L}_{t}(\cdot)$. Thus, a high magnitude $R_{a}$ occurs when the corresponding projections are similar (upper left corner of the figure). The second restriction $R_{b}$ will state that if two instances are temporally correlated, then their projections should be made similar by $\mathcal{L}_{t}(\cdot)$. Thus, an $R_{b}$ with high magnitude happens when the projections are not similar (lower right corner of the figure). In [37] a similar 


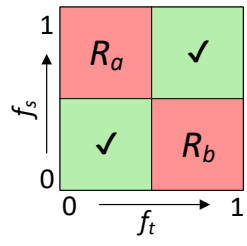

Figure 4: Temporal vs semantic loss terms trade-off.

approach was used to penalize the cost function. However, in our proposal, the cost function is fully differentiable, thus holding a principled loss function formulation capturing temporal information, while retaining all the theoretical guarantees of convergence.

From eq. 7, the adaptive margin $\operatorname{tm}(\cdot)$ enforces instance projections to be separated, which is exactly what must be done when $R_{a}$ is high. On the other hand, when $R_{b}$ is high, projections should be similar. According to this rationale, we define the function $\operatorname{tm}\left(d^{i}, d^{j}\right)$ as:

$$
t m\left(d^{i}, d^{j} ; \boldsymbol{\theta}_{V}, \boldsymbol{\theta}_{T}, \boldsymbol{\theta}_{t e m p}\right)=\left[\min \left(k, R_{a}\left(d^{i}, d^{j}\right)-R_{b}\left(d^{i}, d^{j}\right)\right)\right]_{+},
$$

which combines the two restrictions as a trade-off, through a subtraction. The subtraction is clipped to be in the range $[0, k]$ to avoid early-stagnation during training, due to collapsed margins $(\operatorname{tm}(\cdot) \rightarrow 0)$. The hyper-parameter $k$, thresholds the maximum value of $\operatorname{tm}(\cdot)$ by $k$, between any two instances. In eq. 5 , instances from distinct categories are separated by a high magnitude margin of $m=1$. To avoid provoking overlaps or give contradictory information to the model, $k$ should be smaller than $m$. Lastly, as only the positive part of $t m(\cdot)$ is considered (through the $[x]_{+}$function), if $R_{b}$ dominates $R_{a}$, then we do not want to enforce a margin, but rather make projections similar. In this situation, $\operatorname{tm}(\cdot)$ is replaced by a zero valued margin, instead of setting $\operatorname{tm}(\cdot)=k$. To summarize eq. 8 , an increase in $R_{a}$ directly translates in an increase in the enforced margin $f_{m}$ between the two instances. Conversely, an increase in $R_{b}$ indicates that projections have to be similar, thus resulting in a decrease (towards 0 ) of the margin $f_{m}$.

Finally, the trade-off expressed in Figure 4, is implemented through the following formal definitions:

Definition 2. Restriction $R_{a}$ enforces temporally uncorrelated instances $f_{t}\left(d^{i}, d^{j}\right) \rightarrow 0$, with similar cross-modality projections, $f_{s}\left(d^{i}, d^{j} ; \theta_{V}, \theta_{T}\right) \rightarrow 1$, to be dissimilar, thus having distant projections. This is formalized as:

$$
R_{a}\left(d^{i}, d^{j}\right)=\left(1-f_{t}\left(d^{i}, d^{j} ; \boldsymbol{\theta}_{\text {temp }}\right)\right) \cdot f_{s}\left(d^{i}, d^{j} ; \boldsymbol{\theta}_{V}, \boldsymbol{\theta}_{T}\right) .
$$

Definition 3. Restriction $R_{b}$ enforces temporally correlated instances, $f_{t}\left(d^{i}, d^{j}\right) \rightarrow 1$, with distant cross-modality projections, $f_{s}\left(d^{i}, d^{j} ; \theta_{V}, \theta_{T}\right) \rightarrow 0$, to have similar projections. This is formalized as:

$$
R_{b}\left(d^{i}, d^{j}\right)=f_{t}\left(d^{i}, d^{j} ; \boldsymbol{\theta}_{t e m p}\right) \cdot\left(1-f_{s}\left(d^{i}, d^{j} ; \boldsymbol{\theta}_{V}, \boldsymbol{\theta}_{T}\right)\right) .
$$

4.2.1 Correlations Across Projected Modalities $f_{s}$. Cross-modality similarity $f_{s}$, computed over semantically similar instances of $d^{i}$, is defined as the cross-modal mean of projections' similarities $f_{s}\left(d^{i}, d^{j}\right)=\frac{1}{2} \cdot\left(s\left(x_{V}^{i}, x_{T}^{j}\right)+s\left(x_{T}^{i}, x_{V}^{j}\right)\right)$, where each $s(\cdot, \cdot)$ is first normalized to the $[0,1]$ range, and then the average between the similarities over the two directions is computed. Note that both modalities similarities contribute equally to $f_{s}$.

4.2.2 Temporal Correlations $f_{t}$. Different temporal correlations can be accounted by simply requiring the definition of $f_{t}$, that assesses the temporal correlation between two instances, based on a distribution $\boldsymbol{\theta}_{\text {temp }}$.

Recency-based Correlations. The first approach to temporal correlations assume that an instance $d^{i}$ is temporally correlated to another instance $d^{j}$ if both occur close in time. For temporally distant ones, a non-linear decay is applied. Given two instances $d^{i}$ and $d^{j}$, and their associated timestamps $t^{i}$ and $t^{j}$, respectively, Recency-based correlation is formalized as an exponential function $f_{t}\left(d^{i}, d^{j} ; \theta_{\text {temp }}\right)=e^{-\left|t^{i}-t^{j}\right| \cdot h}$, where $\theta_{\text {temp }}=\{h\}$, with a decay factor $h$ as parameter, to cope with different temporal granularities., and $f_{t}\left(t s^{i}, t s^{j} ; \theta_{\text {temp }}\right)$ maps to the range $\left.] 0,1\right]$. The rationale is to structure instances according to their temporal proximity.

Density-based Correlations. Each category $l$ may be defined by different temporal behaviour along the dataset timespan. Namely, there can be situations in which we want instances that are far apart, but happened at an important/relevant moment ${ }^{1}$, to be structured together in the cross-modal embedding space. Therefore, correlations can be estimated from the temporal distribution $\vec{\phi}_{l}$ of a category $l$. Given $\vec{\phi}_{l}$, we define the temporal density of $l$, at time $t$, as $f_{t}\left(d^{i}, d^{j}\right)=p\left(t^{i} \mid \vec{\phi}_{l}\right) \cdot p\left(t^{j} \mid \vec{\phi}_{l}\right)$, where $p\left(t \mid \vec{\phi}_{l}\right)$ is a Gaussian Kernel Density Estimation (KDE) that for each category $l$, runs through the set of timestamps $T_{l}=\left\{t^{i}: l \in l^{i}, d^{i} \in C\right\}$, with a kernel bandwidth $b$, and where $\boldsymbol{\theta}_{\text {temp }}=\{b\}$. When instances $i$ and $j$ share more than one label, we select the label $l$ that maximizes the corresponding $p\left(t \mid \vec{\phi}_{l}\right)$. We experimented setting a different $b$ for each category, but did not observe significant performance gains (these results are on the supplementary material).

\subsection{Architecture and Training Strategy}

Projection functions $\mathcal{P}_{\boldsymbol{\theta}_{V}}(\cdot)$ and $\mathcal{P}_{\boldsymbol{\theta}_{T}}(\cdot)$ are implemented as neural networks, each with 2 fully connected layers:

$$
\begin{aligned}
& \mathcal{P}_{\theta_{V}}\left(x_{V}^{i}\right)=\tanh \left(\boldsymbol{\theta}_{V_{h 2}} \cdot \tanh \left(\boldsymbol{\theta}_{V_{h 1}} \cdot x_{V}^{i}\right)\right), \\
& \mathcal{P}_{\boldsymbol{\theta}_{T}}\left(x_{T}^{i}\right)=\tanh \left(\boldsymbol{\theta}_{T_{h 2}} \cdot \tanh \left(\boldsymbol{\theta}_{T_{h 1}} \cdot x_{T}^{i}\right)\right),
\end{aligned}
$$

where $\theta_{V}=\left[\theta_{V_{h 1}}, \theta_{V_{h 2}}\right]$ and $\theta_{T}=\left[\theta_{T_{h 1}}, \theta_{T_{h 2}}\right]$ are the network weight matrices, for visual and textual projections, respectively. The first and the second hidden layers, have dimensions 1024 and $D=100$, respectively. Resulting projections are $\ell_{2}$ normalized. The two decoupled network architecture, in which networks are independent but jointly optimized under same loss function $\mathcal{L}$, is common to state-of-the-art cross-modal embedding learning [9, $10,26,45,50]$. Image features $x_{V}^{i} \in \mathbb{R}^{D_{V}=2048}$ are obtained from a pre-trained ResNet-50 [13] CNN on ImageNet [8], in which the softmax layer is removed and feature maps are average pooled. Text features $x_{T}^{i}$ correspond to a TF-IDF bag-of-words representation.

Training and Optimization. We adopt a stochastic triplet sampling strategy, in which to evaluate $\mathcal{L}\left(\boldsymbol{\theta}_{V}, \boldsymbol{\theta}_{T}\right)$, negative instances are sampled directly from mini-batches. In the spirit of $[16,40]$, we

${ }^{1}$ Importance/relevance stems from the scenario which we are trying to model. In this situation we relate importance to peaks of social media reactions to a topic (user posts). 

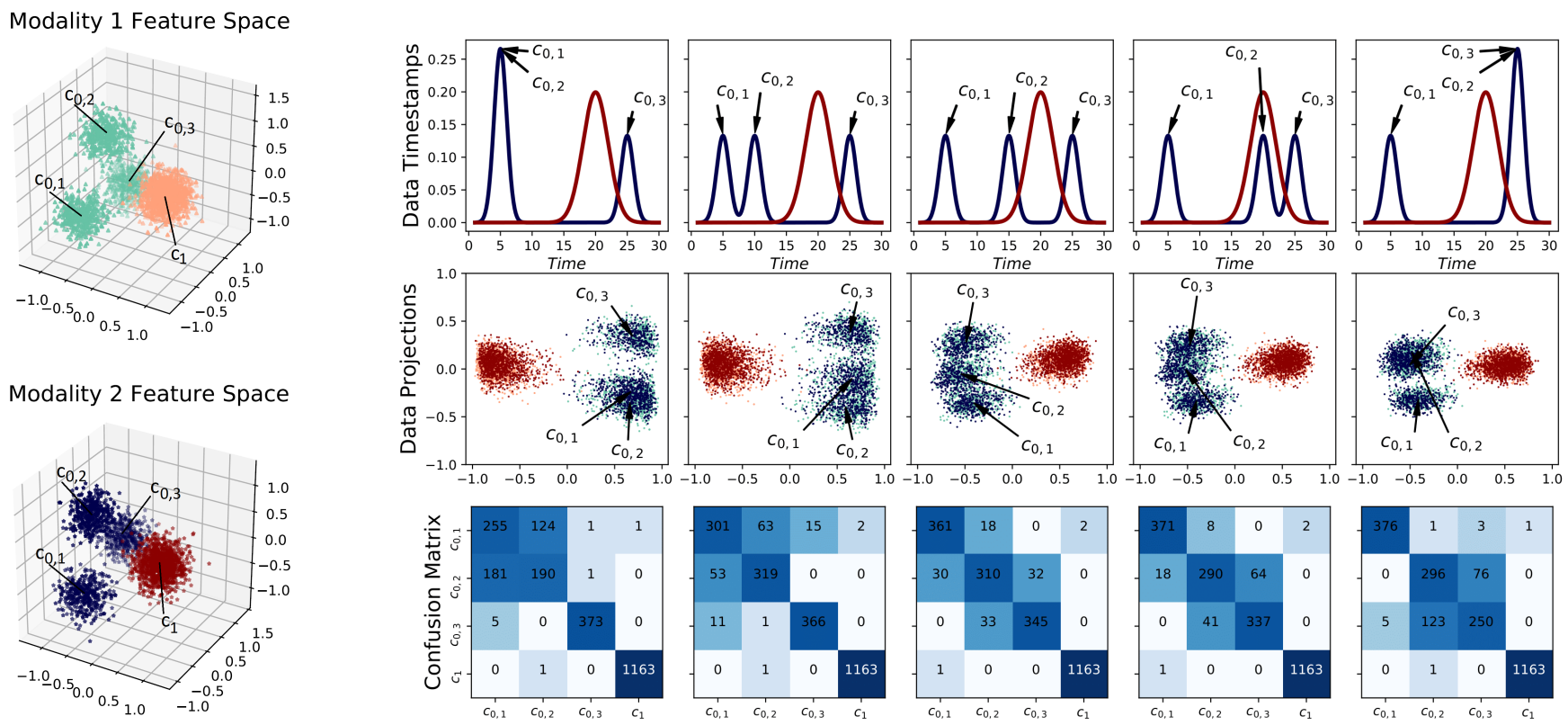

Figure 5: Synthetic experiments: (left) PCA visualization of the 10-D synthetic data; (right) five different temporal distributions of the data depicted, where the top row shows the timestamps of each cluster. The mid row illustrates the projected data and the bottom row quantifies the confusion across clusters of the same categories and different categories.

use all the information contained in a batch. Therefore, for each anchor-positive pair, we considered all the negatives $x_{*}^{n}$. To capture cross-modality correlations, the positive instance of $x_{V}^{i}$ is its modality counterpart (e.g. for each $x_{V}^{i}, x_{V}^{p}=x_{T}^{i}$, and vice-versa). This strategy reduces the complexity of the sampling procedure by an order of magnitude (batch size). At each epoch, all instances are seen by the network. Mini-batch size is set to 64 . The model is optimized using SGD, with 0.9 momentum, a learning rate of $\eta=5 \times 10^{-3}$, a decay of $1 \times 10^{-6}$, with each gradient update step being $\theta_{V}=\theta_{V}-\eta \nabla_{\theta_{V}}\left(\mathcal{L}_{s}+\mathcal{L}_{t}\right)$ and $\boldsymbol{\theta}_{T}=\boldsymbol{\theta}_{T}-\eta \nabla_{\theta_{T}}\left(\mathcal{L}_{s}+\mathcal{L}_{t}\right)$. The model with lower validation error, after 25 epochs, is kept.

\section{CANONICAL ANALYSIS OF TEMPORAL EMBEDDING SPACE}

To analyze how time information can force the organization of the cross-modal embedding and to observe its key properties, we run an experiment with synthetic data, simulating two modalities.

We consider two categories $l_{0}$ and $l_{1}$ with data distributed as observed in Figure 5 (left part). Category $l_{1}$ has one cluster $c_{1}$ and category $l_{0}$ has three clusters, $c_{0,1}, c_{0,2}$ and $c_{0,3}$. Feature vectors $x_{1}^{i}$ and $x_{2}^{i}$ are generated in a 10-dimensional space, by generating a category centroid vector and then adding Gaussian noise.

In this experiment, we wish to observe how data timestamps steer the organization of the embedding space. Hence, instances from different clusters, will have different timestamp distributions as illustrated on the top row of Figure 5. In practice, we fixed the timestamps for category $l_{1}$, and for category $l_{0}$ we fixed the timestamps of the two clusters $c_{0,1}, c_{0,3}$ and shifted the timestamps of cluster $c_{0,2}$ from $c_{0,1}$ towards $c_{0,3}$. This will affect the timestamps distribution of $l_{0}$. Then, for each scenario, we learn a common embedding space with TXS-Adapt-Rec (recency-based temporal correlation), using $D=2$ (for visualization purposes), recency bandwidth $h=0.2$, and evaluate its sensibility to timestamp changes. We expect the data timestamps distribution to affect and guide the structuring of the embedding space. Note that feature vectors are kept fixed throughout all experiments, and only the timestamps of the data samples belonging to cluster $c_{0,1}$ are changed.

When visualizing the learned data projections on the mid row of Figure 5 (for visualization purposes projections are not normalized) we can observe that the cost function $\mathcal{L}_{S}(\cdot)$ forces the separation of the categories $l_{0}$ and $l_{1}$, while the novel adaptive temporal margin $\mathcal{L}_{t}(\cdot)$ steers the temporal organization of the embedding, i.e., the projections of $c_{0,1}, c_{0,2}, c_{0,3}$. The third row of the right part of Figure 5 further confirms this phenomena: the confusion matrices quantify the degree of confusion between embeddings of different clusters, obtained by classifying each instance as belonging to a cluster $-c_{0,1}, c_{0,2}, c_{0,3}, c_{1}$ - using a nearest-neighbor approach.

Starting in the 1st column of right part of Figure 5, we note that clusters $c_{0,1}$ and $c_{0,2}$ overlap in time (day 5). Thus, although these clusters are strongly correlated in time, they are completely separated in their original spaces. Accordingly, a significant degree of confusion between $c_{0,1}$ and $c_{0,2}$ clusters is observed in the confusion matrix, showing that embeddings of instances from those clusters were placed together, as stated by Property 3. Moreover, confusion between the two clusters $c_{0,1}, c_{0,2}$ and the third clusters $c_{0,3}$ is minimal, showing that the corresponding embeddings are distant, as stated by Property 2. Finally, confusion over instances from 
categories $l_{0}$ and $l_{1}$ is minimal, evidencing that the embeddings of instances from distinct categories are clearly separated, as stated by Property 1. The three observations can be further confirmed by visualizing the instance projections (2nd row of Figure 5 (b)), which reveals three distinct clusters: $c_{0,1}, c_{0,2}, c_{0,3}$ and $c_{1}$.

Moving to the other columns of Figure 5, we examine how the cost function $\mathcal{L}_{t}(\cdot)$ steers the organization of the category $l_{0}$ data according to their temporal correlation. Moreover, despite these re-arrangements of the category $l_{0}$, the cost function $\mathcal{L}_{S}(\cdot)$ consistently preserves the separation between the two categories.

\section{EXPERIMENTS}

\subsection{Datasets and Methodology}

To evaluate our model we considered three social media datasets.

NUS-WIDE is a benchmark dataset for cross-modal retrieval [11, $29,47]$, comprising 269,648 image-text pairs annotated with 81 semantic categories. Its temporal dynamic behavior has been observed in prior work [43]. The timestamps of each instance were collected 2 and used to estimate temporal correlations. Each instance belongs to one or more semantic categories. After cleaning invalid data, the dataset is comprised by a total of 169,283 instances.

EdFest and TDF ${ }^{2}$ datasets extends the one from [37], and is comprised by image-text instances from social media. It is organized in two sub-datasets, each covering an event: Edinburgh Festival 2016 and Tour de France 2016. In both datasets instances have strong temporal correlations, as expected from live events than span multiple days. Each instance is annotated with one or more categories, from a total of 13 event-specific semantic categories. A total of 2000 (EdFest) and 955 (TDF) annotated pairs are available.

Protocol, Metrics and Baselines. Following the cross-modal retrieval literature $[10,30,45,47,50]$, we consider the tasks of retrieving text with images (I-T) and vice-versa (T-I). Each query consists of an image $x_{V}^{i}$ or text $x_{T}^{i}$, and an instance is deemed relevant if it belongs to the query category. Retrieval results are evaluated with mean Average Precision ( $m A P @ K)$ and $K=50[10,37]$.

For all datasets, $90 \%$ of the data is used for training, $10 \%$ for testing, and $15 \%$ of training data for validation. Models' granularity is set to days for EdFest/TDF and years for NUS-WIDE. We evaluate TXS-Adapt with recency-based (TXS-Adapt-Recency), and category-based temporal correlations (TXS-Adapt-Density). Additionally, we consider a static variant Static XS in which temporal information is discarded (i.e. $\mathcal{L}_{t}=0$ ). Recency bandwidth is set to $h=0.5$ for EdFest/TDF and to $h=0.2$ to NUS-WIDE, and KDE bandwidth $b=1$. We adopt a non-temporal linear embedding space learning baseline, CCA [30], and state-of-the-art neural baselines: Deep-CCA [1, 50], ACMR [45]. Finally, we also consider a temporal recency-based baseline TempXNet [37]. Both $x_{V}^{i}$ and $x_{T}^{i}$ representations are the same for all methods.

\subsection{Results and Discussion}

Temporal Structure-Preserving Embeddings. First, to understand how TXS-Adapt structures data w.r.t. temporal correlations, for each query $x_{*}^{i}$ (image or text) with timestamp $t^{i}$, we evaluate the temporal variation between $t^{i}$ and the 5 closest neighbors (instances

\footnotetext{
${ }^{2}$ Available here: https://novasearch.org/temporal-cross-modal-embeddings/.
}

Table 1: Temporal variation over top-5 nearest neighbors.

\begin{tabular}{l|cccc}
\hline & \multicolumn{2}{|c}{$\mathrm{I}-\mathrm{T}$} & \multicolumn{2}{c}{$\mathrm{T}-\mathrm{I}$} \\
& $\boldsymbol{\Delta}_{\boldsymbol{t}}$ & $\boldsymbol{\Delta}_{\boldsymbol{m} \boldsymbol{s e}}$ & $\boldsymbol{\Delta}_{\boldsymbol{t}}$ & $\boldsymbol{\Delta}_{\boldsymbol{m} \boldsymbol{s} \boldsymbol{e}}$ \\
\hline Method & \multicolumn{4}{c}{ EdFest } \\
\hline Static XS $\left(\mathcal{L}_{t}=0\right)$ & 8.35 & 640.8 & 7.96 & 608.8 \\
TempXNet [37] & 8.11 & 615.2 & 7.98 & 609.6 \\
TXS-Adapt-Recency & $\mathbf{7 . 7 2}$ & $\mathbf{5 8 6 . 3}$ & $\mathbf{7 . 6 7}$ & $\mathbf{5 8 4 . 7}$ \\
TXS-Adapt-Density & 7.75 & 587.2 & 7.76 & 593.3 \\
\hline & \multicolumn{5}{|c}{ TDF } \\
\hline Static XS $\left(\mathcal{L}_{t}=0\right)$ & 7.05 & 543.9 & 7.1 & 564.2 \\
TempXNet [37] & 6.81 & 527.9 & 6.76 & 526.4 \\
TXS-Adapt-Recency & $\mathbf{6 . 5 2}$ & $\mathbf{4 9 8 . 1}$ & $\mathbf{6 . 6 1}$ & $\mathbf{5 1 2 . 0}$ \\
TXS-Adapt-Density & 6.80 & 528.6 & 6.68 & 519.2 \\
\hline
\end{tabular}

of the opposite modality), sharing a semantic category, from the training set. Table 1 shows the results, where $\Delta_{t}$ is the average of the differences (absolute value) between each neighbouring instance timestamp $t^{i}$ and the timestamp of the query, and $\Delta_{m s e}$ the average of the mean-squared-error of these differences, over all queries. First, we can see that the static approach, which neglects time, has a high $\Delta_{t}$ and $\Delta_{m s e}$. This is expected as no attempt to structure temporally correlated instances in the same neighborhood (Property 3) is made. This evidences the main characteristic of a temporal cross-modal embedding. Namely, by focusing on the results of TXS-Adapt-Recency, we observe that on both datasets, neighbours are closer to the query (temporal distance). TXS-Adapt-Density, despite employing a different measure of temporal correlation (density curve), still manages to structure instances based on temporal proximity better than the static baseline. This suggests that some densities may have a single mode. In fact, if all the densities had a single mode, then TXS-Adapt-Density models could be seen as a smoothed version of TXS-Adapt-Recency. Even though TempXNet outperforms Static XS, our proposed TXS-Adapt model achieves significantly better structuring w.r.t. temporal correlations.

Cross-modal Retrieval. We evaluate the proposed temporal embedding in the task of cross-modal retrieval, on the test set. In Table 2 we observe that TXS-Adapt is consistently effective across the three datasets, outperforming all the other baselines, on both tasks. Namely, for EdFest, TXS-Adapt-Recency outperformed TXSAdapt-Density and all the baselines. For TDF, TXS-Adapt-Density was the best performing method. On NUS-WIDE, both TXS-Adapt variants achieve similar performance. The fact that better performance is obtained when projections are structured according to temporal and semantic correlations, and that different variants yield different performances, reflects the existence of distinct temporal distributions, underlying each dataset. We recall that both EdFest and TDF are dynamic collections, thus, data is expected to have strong, but different, temporal correlations. For collections with single mode distribution events (e.g. shows, stages, etc.), temporal proximity is adequate. This is consistent with TXS-Adapt-Recency being the best method on EdFest. For collections with multiple modes and recurring events, density based temporal distributions are more adequate, which again, is consistent with TXS-AdaptDensity being the best method on TDF. For NUS-WIDE, which can 


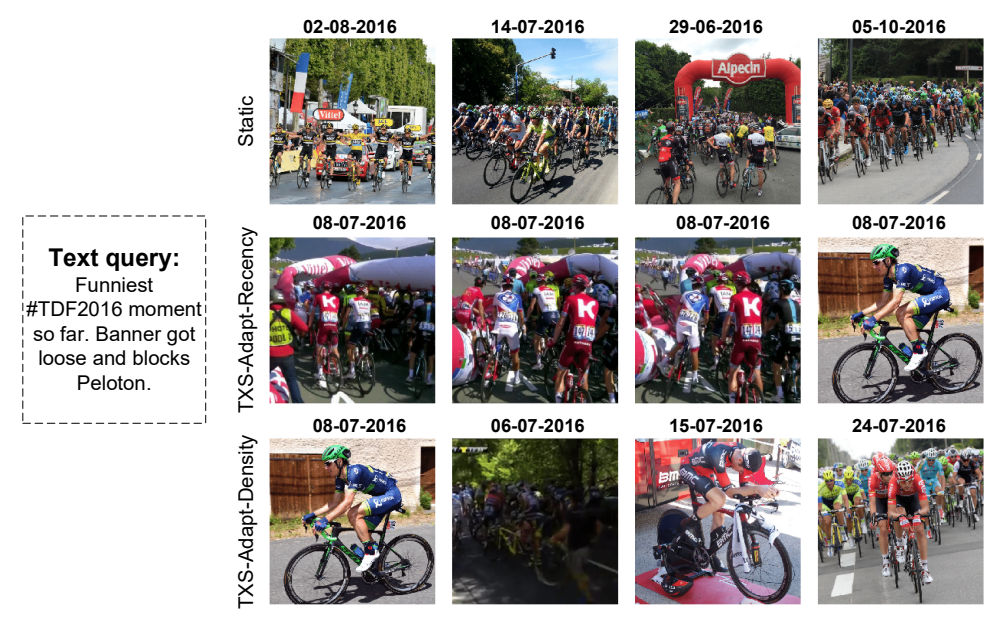

Figure 6: Qualitative comparison between different embeddings for summarizing the same text query, on TDF dataset (T-I direction).

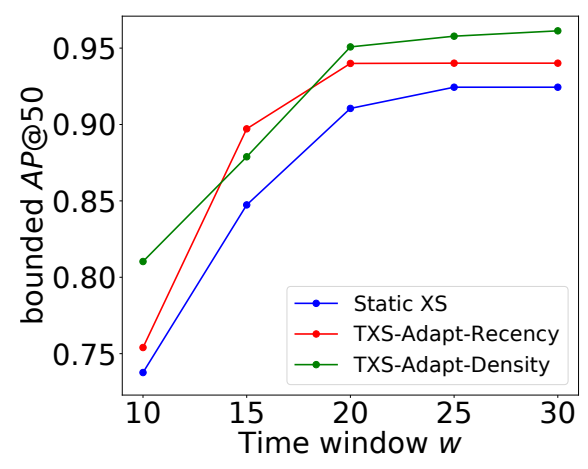

Figure 7: Bounded Average Precision, for the query shown in Figure 6 (at the left), on different time windows. For each $w$, an image is only relevant if it belongs to the same category and is not more than $w$ days apart from the query timestamp. be regarded as a photo album, temporal correlations are expected to be minimal, and performances of TXS-Adapt variants are more uniform.

Summarization. In this section we showcase a direct application of the proposed embedding to the task of summarization: find a set of images that summarize a topic (text). To this extent, we gave the model a text as input, and directly exploited the geometry of the temporal embedding space to summarize the topic conveyed by that text. Figure 6 shows the produced summaries, where each line represents the nearest neighbors to the query. The first summary was obtained with a static embedding which neglects time, as such, content is grouped without any temporal structuring. In the second, as TXS-Adapt-Recency was used, images are all close in time. The third summary, used TXS-Adapt-Density, comprises images from multiple days, which unlike TXS-Adapt-Recency, provides a wider coverage in terms of obtaining images from distinct instants.

To further understand the behavior of each variant, in Figure 7 we show the performance for the same query, when we restrict the relevant images to the ones that are only $w$ days apart from the query. The main observation is that the most suitable correlation depends on user requirements. Namely, despite TXS-Adapt-Recency being outperformed by Static XS in TDF (Table 2), for this query it not only outperformed Static XS but also created the most precise summary with the images depicting the moment described by the text. In contrast, TXS-Adapt-Density provided a more diverse summary. Note that no manual filtering, or ad-hoc procedures were performed to obtain the following summaries. The structure of the summary emerges directly from each type of embedding. For additional analysis and examples check the supplementary material.

\section{CONCLUSIONS}

Adaptive temporal margins. In this paper we proposed TXSAdapt, an adaptive maximum-margin temporal cross-modal embedding space learning method, with a new theoretical formulation of triplet loss where static margins were replaced by temporal margin.
Table 2: Cross-modal retrieval performance (mAP@50) on the EdFest, TDF and NUS-WIDE datasets.

\begin{tabular}{|c|c|c|c|c|c|c|}
\hline \multirow[b]{2}{*}{ Method } & \multicolumn{2}{|c|}{ EdFest } & \multicolumn{2}{|c|}{ TDF } & \multicolumn{2}{|c|}{ NUS-WIDE } \\
\hline & $\mathrm{I}-\mathrm{T}$ & T-I & $\mathrm{I}-\mathrm{T}$ & $\mathrm{T}-\mathrm{I}$ & $\mathrm{I}-\mathrm{T}$ & T-I \\
\hline CCA [30] & 38.4 & 43.5 & 59.4 & 61.1 & 71.6 & 63.1 \\
\hline Deep-CCA [50] & 46.4 & 50.9 & 62.8 & 66.0 & 73.9 & 76.1 \\
\hline ACMR [45] & 69.5 & 56.7 & 66.3 & 70.7 & 75.9 & 73.7 \\
\hline TempXNet [37] & 72.9 & 62.9 & 71.3 & 70.0 & 77.7 & 77.1 \\
\hline$\overline{\text { Static }} \overline{\mathrm{XS}}\left(\overline{\mathcal{L}_{t}}=\overline{0}\right)$ & $-\overline{7} . \overline{4}$ & $\overline{62} . \overline{9}$ & $\overline{73.0}$ & $\overline{73.1}$ & $-\overline{7 \overline{7}} . \overline{8}$ & $\overline{77 . \overline{0}}$ \\
\hline$\overline{\mathrm{T} X S} \overline{\mathrm{A}}-\overline{\mathrm{A}} \mathrm{-}-\bar{p} \mathrm{t}-\mathrm{Recency}$ & $\overline{7} \overline{3} . \overline{6}$ & $\overline{65 . \overline{4}}$ & $\overline{7} 2 . \overline{4}$ & $\overline{71.6}$ & $-\overline{8} . \overline{6}$ & $\overline{78.0}$ \\
\hline TXS-Adapt-Density & 73.4 & 64.0 & 73.7 & 73.3 & 78.5 & 78.0 \\
\hline
\end{tabular}

Temporal embeddings. The redesign of the triplet loss function, enables the creation of an embedding space that is temporally structured by an adaptive margin function, modeling data dynamics through temporal constraints.

Applicability to other domains. Experiments showed the versatility and usefulness of temporal cross-modal embeddings, with the embedding geometry capturing the temporal traits of multimodal (visual and textual) data. These same advantages are key to many other domains, including face age detection, exploration of multimodal historical archives, trend analysis, timeline summarization, egocentric video summarization, and others.

\section{ACKNOWLEDGMENTS}

This work has been partially funded by the iFetch project, Ref. 45920, co-financed by ERDF, COMPETE 2020, NORTE 2020 and FCT under CMU Portugal, by the CMU Portugal GoLocal project Ref. CMUP-ERI/TIC/0046/2014, by the H2020 ICT COGNITUS project with the grant agreement $\mathrm{n}^{\circ} 687605$ and by the FCT project NOVA LINCS Ref. UID/CEC/04516/2019. We also gratefully acknowledge the support of NVIDIA Corporation with the donation of the GPUs used for this research. 


\section{REFERENCES}

[1] Galen Andrew, Raman Arora, Jeff Bilmes, and Karen Livescu. 2013. Deep Canonical Correlation Analysis. In Proceedings of the 30th International Conference on International Conference on Machine Learning - Volume 28 (ICML'13). JMLR.org, III-1247-III-1255. http://dl.acm.org/citation.cfm?id=3042817.3043076

[2] Stanislaw Antol, Aishwarya Agrawal, Jiasen Lu, Margaret Mitchell, Dhruv Batra, C. Lawrence Zitnick, and Devi Parikh. 2015. VQA: Visual Question Answering. In International Conference on Computer Vision (ICCV).

[3] T. Baltrušaitis, C. Ahuja, and L. Morency. 2019. Multimodal Machine Learning A Survey and Taxonomy. IEEE Transactions on Pattern Analysis and Machine Intelligence 41, 2 (Feb 2019), 423-443. https://doi.org/10.1109/TPAMI.2018.2798607

[4] Yoshua Bengio, Réjean Ducharme, Pascal Vincent, and Christian Janvin. 2003. A Neural Probabilistic Language Model. 7. Mach. Learn. Res. 3 (March 2003), 1137-1155. http://dl.acm.org/citation.cfm?id=944919.944966

[5] David M. Blei and John D. Lafferty. 2006. Dynamic Topic Models. In Proceedings of the 23rd International Conference on Machine Learning (ICML '06). ACM, New York, NY, USA, 113-120. https://doi.org/10.1145/1143844.1143859

[6] Sarath Chandar, Mitesh M. Khapra, Hugo Larochelle, and Balaraman Ravindran. 2016. Correlational Neural Networks. Neural Comput. 28, 2 (Feb. 2016), 257-285. https://doi.org/10.1162/NECO_a_00801

[7] Weihua Chen, Xiaotang Chen, Jianguo Zhang, and Kaiqi Huang. 2017. Beyond triplet loss: a deep quadruplet network for person re-identification. In Proceedings of the IEEE Conference on Computer Vision and Pattern Recognition. 403-412.

[8] J. Deng, W. Dong, R. Socher, L. Li, Kai Li, and Li Fei-Fei. 2009. ImageNet: A largescale hierarchical image database. In 2009 IEEE Conference on Computer Vision and Pattern Recognition. 248-255. https://doi.org/10.1109/CVPR.2009.5206848

[9] Mengdi Fan, Wenmin Wang, Peilei Dong, Liang Han, Ronggang Wang, and Ge Li. 2017. Cross-media Retrieval by Learning Rich Semantic Embeddings of Multimedia. In Proceedings of the 2017 ACM on Multimedia Conference (MM '17) ACM, New York, NY, USA, 1698-1706. https://doi.org/10.1145/3123266.3123369

[10] Fangxiang Feng, Xiaojie Wang, and Ruifan Li. 2014. Cross-modal Retrieval with Correspondence Autoencoder. In Proceedings of the 22Nd ACM International Conference on Multimedia (MM '14). ACM, New York, NY, USA, 7-16. https: //doi.org/10.1145/2647868.2654902

[11] Yunchao Gong, Oifa Ke, Michael Isard, and Svetlana Lazebnik. 2014. A MultiView Embedding Space for Modeling Internet Images, Tags, and Their Semantics. Int. F. Comput. Vision 106, 2 (Jan. 2014), 210-233. https://doi.org/10.1007/ s11263-013-0658-4

[12] William L Hamilton, Jure Leskovec, and Dan Jurafsky. 2016. Diachronic word embeddings reveal statistical laws of semantic change. arXiv preprint arXiv:1605.09096 (2016)

[13] Kaiming He, Xiangyu Zhang, Shaoging Ren, and Jian Sun. 2016. Deep Residual Learning for Image Recognition. In The IEEE Conference on Computer Vision and Pattern Recognition (CVPR).

[14] Xiangteng He, Yuxin Peng, and Liu Xie. 2019. A New Benchmark and Approach for Fine-Grained Cross-Media Retrieval. In Proceedings of the 27th ACM International Conference on Multimedia (MM '19). Association for Computing Machinery, New York, NY, USA, 1740-1748. https://doi.org/10.1145/3343031.3350974

[15] Ralf Herbrich, Thore Graepel, and Klaus Obermayer. 2000. Large Margin Rank Boundaries for Ordinal Regression. In Advances in Large Margin Classifiers, P. J. Bartlett, B. Schölkopf, D. Schuurmans, and A. J. Smola (Eds.). MIT Press, 115-132.

[16] Alexander Hermans*, Lucas Beyer*, and Bastian Leibe. 2017. In Defense of the Triplet Loss for Person Re-Identification. arXiv preprint arXiv:1703.07737 (2017).

[17] D. Hu, X. Li, and X. Lu. 2016. Temporal Multimodal Learning in Audiovisual Speech Recognition. In 2016 IEEE Conference on Computer Vision and Pattern Recognition (CVPR). 3574-3582. https://doi.org/10.1109/CVPR.2016.389

[18] Andrej Karpathy and Li Fei-Fei. 2017. Deep Visual-Semantic Alignments for Generating Image Descriptions. IEEE Trans. Pattern Anal. Mach. Intell. 39, 4 (April 2017), 664-676. https://doi.org/10.1109/TPAMI.2016.2598339

[19] Gunhee Kim and Eric P. Xing. 2013. Time-sensitive Web Image Ranking and Retrieval via Dynamic Multi-task Regression. In Proceedings of the Sixth ACM International Conference on Web Search and Data Mining (WSDM '13). ACM, New York, NY, USA, 163-172. https://doi.org/10.1145/2433396.2433417

[20] Brian Kulis et al. 2013. Metric learning: A survey. Foundations and Trends ${ }^{\circledR}$ in Machine Learning 5, 4 (2013), 287-364.

[21] Jey Han Lau, Nigel Collier, and Timothy Baldwin. 2012. On-line Trend Analysis with Topic Models: \#twitter Trends Detection Topic Model Online. In Proceedings of COLING 2012. The COLING 2012 Organizing Committee, 1519-1534. http: //aclweb.org/anthology/C12-1093

[22] Jey Han Lau, Nigel Collier, and Timothy Baldwin. 2012. On-line Trend Analysis with Topic Models: \#twitter Trends Detection Topic Model Online. In COLING.

[23] Yu-Ying Liu, Shuang Li, Fuxin Li, Le Song, and James M Rehg. 2015. Efficient Learning of Continuous-Time Hidden Markov Models for Disease Progression. In Advances in Neural Information Processing Systems 28, C. Cortes, N. D. Lawrence, D. D. Lee, M. Sugiyama, and R. Garnett (Eds.). Curran Associates, Inc., 3600-3608.

[24] Jiasen Lu, Jianwei Yang, Dhruv Batra, and Devi Parikh. 2016. Hierarchical Question-image Co-attention for Visual Question Answering. In Proceedings of the 30th International Conference on Neural Information Processing Systems
(NIPS'16). Curran Associates Inc., USA, 289-297. http://dl.acm.org/citation.cfm? id $=3157096.3157129$

[25] Flávio Martins, João Magalhães, and Jamie Callan. 2019. Modeling Temporal Evidence from External Collections. In Proceedings of the Twelfth ACM International Conference on Web Search and Data Mining (WSDM '19). Association for Computing Machinery, New York, NY, USA, 159-167. https://doi.org/10.1145/ 3289600.3290966

[26] Jiquan Ngiam, Aditya Khosla, Mingyu Kim, Juhan Nam, Honglak Lee, and Andrew Y. Ng. 2011. Multimodal Deep Learning. In Proceedings of the 28th International Conference on International Conference on Machine Learning (ICML'11). Omnipress, USA, 689-696. http://dl.acm.org/citation.cfm?id=3104482.3104569

[27] Yingwei Pan, Yehao Li, Ting Yao, Tao Mei, Houqiang Li, and Yong Rui. 2016. Learning Deep Intrinsic Video Representation by Exploring Temporal Coherence and Graph Structure. In Proceedings of the Twenty-Fifth International foint Conference on Artificial Intelligence (IfCAI'16). AAAI Press, 3832-3838. http://dl.acm.org/citation.cfm?id=3061053.3061155

[28] Yuxin Peng, Xin Huang, and Jinwei Qi. 2016. Cross-media Shared Representation by Hierarchical Learning with Multiple Deep Networks. In Proceedings of the Twenty-Fifth International foint Conference on Artificial Intelligence (IfCAI'16). AAAI Press, 3846-3853. http://dl.acm.org/citation.cfm?id=3061053.3061157

[29] Y. Peng, J. Qi, X. Huang, and Y. Yuan. 2018. CCL: Cross-modal Correlation Learning With Multigrained Fusion by Hierarchical Network. IEEE Transactions on Multimedia 20, 2 (Feb 2018), 405-420. https://doi.org/10.1109/TMM.2017. 2742704

[30] Nikhil Rasiwasia, Jose Costa Pereira, Emanuele Coviello, Gabriel Doyle, Gert R.G. Lanckriet, Roger Levy, and Nuno Vasconcelos. 2010. A New Approach to Cross-modal Multimedia Retrieval. In Proceedings of the 18th ACM International Conference on Multimedia (MM '10). ACM, New York, NY, USA, 251-260. https://doi.org/10.1145/1873951.1873987

[31] Soheil Rayatdoost. 2017. Cross-modality interaction between EEG signals and facial expression. In ICMI.

[32] Takeshi Sakaki, Makoto Okazaki, and Yutaka Matsuo. 2010. Earthquake Shakes Twitter Users: Real-time Event Detection by Social Sensors. In Proceedings of the 19th International Conference on World Wide Web (WWW'10). ACM, New York, NY, USA, 851-860. https://doi.org/10.1145/1772690.1772777

[33] Adam Santoro, David Raposo, David G Barrett, Mateusz Malinowski, Razvan Pascanu, Peter Battaglia, and Timothy Lillicrap. 2017. A simple neural network module for relational reasoning. In Advances in Neural Information Processing Systems 30, I. Guyon, U. V. Luxburg, S. Bengio, H. Wallach, R. Fergus, S. Vishwanathan, and R. Garnett (Eds.). Curran Associates, Inc., 4967-4976. http://papers.nips.cc/ paper/7082-a-simple-neural-network-module-for-relational-reasoning.pdf

[34] Manos Schinas, Symeon Papadopoulos, Georgios Petkos, Yiannis Kompatsiaris, and Pericles A. Mitkas. 2015. Multimodal Graph-Based Event Detection and Summarization in Social Media Streams. In Proceedings of the 23rd ACM International Conference on Multimedia (MM '15). Association for Computing Machinery, New York, NY, USA, 189-192. https://doi.org/10.1145/2733373.2809933

[35] F. Schroff, D. Kalenichenko, and J. Philbin. 2015. FaceNet: A unified embedding for face recognition and clustering. In 2015 IEEE Conference on Computer Vision and Pattern Recognition (CVPR). 815-823. https://doi.org/10.1109/CVPR.2015.7298682

[36] David Semedo. 2020. Bridging Vision and Language over Time with Neural Crossmodal Embeddings. Ph.D. Dissertation. https://run.unl.pt/handle/10362/101656

[37] David Semedo and Joao Magalhaes. 2018. Temporal Cross-Media Retrieval with Soft-Smoothing. In 2018 ACM Multimedia Conference on Multimedia Conference (MM '18). ACM, New York, NY, USA, 1038-1046. https://doi.org/10.1145/3240508. 3240665

[38] David Semedo and Joao Magalhaes. 2019. Cross-Modal Subspace Learning with Scheduled Adaptive Margin Constraints. In Proceedings of the 27th ACM International Conference on Multimedia (MM '19). Association for Computing Machinery, New York, NY, USA, 75-83. https://doi.org/10.1145/3343031.3351030

[39] David Semedo and Joao Magalhaes. 2019. Diachronic Cross-Modal Embeddings. In Proceedings of the 27th ACM International Conference on Multimedia (MM '19). Association for Computing Machinery, New York, NY, USA, 2061-2069. https://doi.org/10.1145/3343031.3351036

[40] Hyun Oh Song, Yu Xiang, Stefanie Jegelka, and Silvio Savarese. 2016. Deep Metric Learning via Lifted Structured Feature Embedding. In Computer Vision and Pattern Recognition (CVPR).

[41] Nitish Srivastava, Elman Mansimov, and Ruslan Salakhudinov. 2015. Unsupervised Learning of Video Representations using LSTMs. In Proceedings of the 32nd International Conference on Machine Learning (Proceedings of Machine Learning Research), Francis Bach and David Blei (Eds.), Vol. 37. PMLR, Lille, France, 843-852. http://proceedings.mlr.press/v37/srivastava15.html

[42] Mikalai Tsytsarau, Themis Palpanas, and Malu Castellanos. 2014. Dynamics of News Events and Social Media Reaction. In Proceedings of the 20th ACM SIGKDD International Conference on Knowledge Discovery and Data Mining (KDD '14). ACM, New York, NY, USA, 901-910. https://doi.org/10.1145/2623330.2623670

[43] Tiberio Uricchio, Lamberto Ballan, Marco Bertini, and Alberto Del Bimbo. 2013. Evaluating Temporal Information for Social Image Annotation and Retrieval. In Image Analysis and Processing - ICIAP 2013, Alfredo Petrosino (Ed.). Springer 
Berlin Heidelberg, Berlin, Heidelberg, 722-732.

[44] Oriol Vinyals, Alexander Toshev, Samy Bengio, and Dumitru Erhan. 2015. Show and Tell: A Neural Image Caption Generator. In The IEEE Conference on Computer Vision and Pattern Recognition (CVPR).

[45] Bokun Wang, Yang Yang, Xing Xu, Alan Hanjalic, and Heng Tao Shen. 2017. Adversarial Cross-Modal Retrieval. In Proceedings of the 2017 ACM on Multimedia Conference (MM '17). ACM, New York, NY, USA, 154-162. https://doi.org/10. $1145 / 3123266.3123326$

[46] K. Wang, R. He, L. Wang, W. Wang, and T. Tan. 2016. Joint Feature Selection and Subspace Learning for Cross-Modal Retrieval. IEEE Transactions on Pattern Analysis and Machine Intelligence 38, 10 (Oct 2016), 2010-2023. https://doi.org/ 10.1109/TPAMI.2015.2505311

[47] Kaiye Wang, Qiyue Yin, Wei Wang, Shu Wu, and Liang Wang. 2016. A Comprehensive Survey on Cross-modal Retrieval. CoRR abs/1607.06215 (2016)

[48] William Yang Wang, Yashar Mehdad, Dragomir R. Radev, and Amanda Stent 2016. A Low-Rank Approximation Approach to Learning Joint Embeddings of News Stories and Images for Timeline Summarization. In Proceedings of the 2016 Conference of the North American Chapter of the Association for Computational Linguistics: Human Language Technologies. Association for Computational Linguistics, San Diego, California, 58-68. https://doi.org/10.18653/v1/N16-1008

[49] Xin Wang, Yuan-Fang Wang, and William Yang Wang. 2018. Watch, Listen, and Describe: Globally and Locally Aligned Cross-Modal Attentions for Video
Captioning. In Proceedings of the 2018 Conference of the North American Chapter of the Association for Computational Linguistics: Human Language Technologies.

[50] F. Yan and K. Mikolajczyk. 2015. Deep correlation for matching images and text. In 2015 IEEE Conference on Computer Vision and Pattern Recognition (CVPR). 3441-3450. https://doi.org/10.1109/CVPR.2015.7298966

[51] Rui Yan, Xiaojun Wan, Mirella Lapata, Wayne Xin Zhao, Pu-Jen Cheng, and Xiaoming Li. 2012. Visualizing Timelines: Evolutionary Summarization via Iterative Reinforcement between Text and Image Streams. In Proceedings of the 21st ACM International Conference on Information and Knowledge Management (CIKM '12). Association for Computing Machinery, New York, NY, USA, 275-284. https://doi.org/10.1145/2396761.2396799

[52] Xitong Yang, Palghat Ramesh, Radha Chitta, Sriganesh Madhvanath, Edgar A. Bernal, and Jiebo Luo. 2017. Deep Multimodal Representation Learning from Temporal Data. 2017 IEEE Conference on Computer Vision and Pattern Recognition (CVPR) (2017), 5066-5074.

[53] Xitong Yang, Palghat Ramesh, Radha Chitta, Sriganesh Madhvanath, Edgar A. Bernal, and Jiebo Luo. 2017. Deep Multimodal Representation Learning from Temporal Data. In CVPR. IEEE Computer Society, 5066-5074.

[54] Zijun Yao, Yifan Sun, Weicong Ding, Nikhil Rao, and Hui Xiong. 2018. Dynamic Word Embeddings for Evolving Semantic Discovery. In Proceedings of the Eleventh ACM International Conference on Web Search and Data Mining (WSDM '18). ACM, New York, NY, USA, 673-681. https://doi.org/10.1145/3159652.3159703 